\title{
Effect of Lithium Doping on the Properties L-Citrulline Oxalate Monohydrate Nonlinear Optical Crystals
}

\author{
Vallath Govindan Sreevalsa ${ }^{*}$, Sankaran Jayalekshmi \\ Division for Research in Advanced Materials,Department of Physics, \\ Cochin University of Science and Technology, Cochi, India \\ Email: ${ }^{*}$ sreevalsavg@gmail.com, jayalekshmi@cusat.ac.in
}

Received August 5, 2012; revised September 12, 2012; accepted September 22, 2012

\begin{abstract}
Single crystals of pure and $\mathrm{Li}^{+}$doped L-citrulline oxalate (LCO) crystals are grown successfully by slow evaporation technique. The effect of lithium doping on crystal properties has been studied. The samples are characterized by X-ray powder diffraction, TGA, FTIR, Raman and UV-Vis spectroscopic techniques. The presence of lithium in $\mathrm{Li}^{+}$doped LCO crystals is estimated using AES. The thermal stability and the optical transparency region are found to be enhanced for the doped samples. The second harmonic generation efficiency of the doped crystal has improved considerably compared to pure L-citrulline oxalate. Lithium doping can hence be considered as a simple and advantageous technique to improve the thermal, optical and non linear optical (NLO) properties of the LCO single crystals.
\end{abstract}

Keywords: Doping; X-Ray Diffraction; Growth from Solutions; Single Crystal Growth; Nonlinear Optic Material

\section{Introduction}

Nonlinear optical (NLO) organic materials are of current interest owing to their wide applications in the domain of optoelectronics and photonics. Nonlinear optical crystals find applications in various fields due to their high hyperpolarisability. Amino acids are potential candidates for optical second harmonic generation (SHG) because they contain chiral carbon atoms and crystallize in noncentro symmetric space groups. Amino acid complexes show good nonlinear response and are promising candidates for coherent blue-green laser generation and frequency doubling applications [1-6]. Most of the organic NLO crystals are constituted by weak van der Waals bonds and hydrogen bonds. So, they have poor mechanical and thermal properties. Doping the organic complexes with inorganic elements affects most of the properties positively. Over the past decade, efforts have been made to characterize the structure, and NLO behavior of materials, with a view to the designing of new NLO materials. One of the most suitable models is the theory of anionic group, which is based on two fundamental assumptions. The first is based on the fact that the anionic groups in the crystal structure are mainly responsible for the NLO properties and the observed NLO susceptibility tensor of a crystal is the geometrical superposition of the microscopic NLO susceptibilities of the anionic groups. The second one is that the microscopic second-order sus-

\footnotetext{
"Corresponding author.
}

ceptibilities of the anionic groups can be calculated from the localized molecular orbital of the group by approximation methods used in quantum chemistry. Some researchers are also of the opinion that the anion group in the crystal is not the only factor making contributions to the nonlinearity of the crystal. The cations, especially the cations with large radius and changeable electron-cloud, also play some significant role in the production of nonlinearity in the crystal [7].

It has been reported that SHG efficiency can be greatly enhanced by altering the molecular alignment through inclusion complexation [8] literature survey on lithium doped materials show that many of the inherent properties of amino acid based materials can be modified by the doping of lithium ions. Lithium is mainly selected to study the effect of cations on the nonlinearity of crystals, because lithium exists at the upper-left of the periodic table of elements and has the strongest polarity among the metal cations.

Attempts to dope L-alanine with lithium [9], L-histidine acetate [10] and Larginine acetate [11] with metals like copper and magnesium have resulted in the betterment of the properties of the parent crystal. Lithium based materials find applications in the fields of solid state rechargeable batteries, optical and acoustic devices and as photorefractive materials. In the present work, a new organic crystal, L-citrulline oxalate [LCO], showing moderate nonlinearity $[12,13]$, has been grown by the simple slow evaporation technique. Doping the crystal 
with lithium enhances the nonlinear optical properties and improves the optical and thermal properties. The present work highlights the advantageous effects of lithium doping on the optical and nonlinear properties of L-citrulline oxalate crystals.

\section{Experimental}

Aqueous solutions of L-citrulline and oxalic acid were mixed in equimolar ratio to get L-citrulline oxalate (LCO) as mentioned in an earlier report $[12,13]$. For growing the doped crystals, $2 \mathrm{~mol} \%$ of lithium sulphate was added to the LCO solution. The seed crystals of both pure and lithium doped L-citrulline oxalate (LCOL) were obtained by slow evaporation of the respective solutions in doubly distilled water at ambient temperature. Good quality crystals were harvested in a period of 30 days Figure 1 shows the photograph of the as grown doped crystals. The crystals have been found to be transparent and free from defects.

\section{Characterization}

The synthesized crystals were structurally characterized by powder XRD, using Bruker D8 advance diffractometer with $\mathrm{CuK} \alpha$ radiation $(\lambda=1.5418 \AA)$. The infra red spectrum was recorded using the AVTAR 370 DTGS FT-IR spectrophotometer to analyze the bonding nature in the crystal. The Raman spectrum was also taken. The UV-Vis-absorption spectrum was taken using JASCO V570 UV-Vis spectrophotometer. TGA studies were carried out using Q50 V20.10 in an atmosphere of nitrogen at a heating rate of $20^{\circ} \mathrm{C}$ in the temperature range $30^{\circ} \mathrm{C}-800^{\circ} \mathrm{C}$. The AES spectrum was also taken to confirm the presence of the dopant in the sample. The second harmonic generation efficiency of the samples was determined by the Kurtz Perry powder technique.

\section{Results and Discussion}

L-citrulline oxalate has already been identified as a promising organic nonlinear optical material $[12,13]$. The present work reveals the influence of lithium doping on the growth and properties of LCO single crystals.

\subsection{Powder X-Ray Diffraction}

$\mathrm{X}$-ray powder diffraction technique was used to ascertain

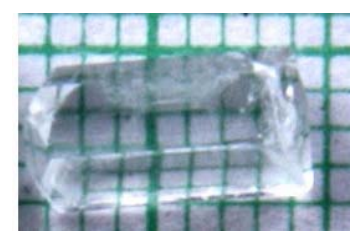

Figure 1. Photograph of as grown $\mathrm{Li}^{+}$doped LCO crystals. the purity of the grown crystals. The XRD patterns of both samples are shown in Figure 2.

From the powder X-ray diffraction studies of $\mathrm{Li}^{+}$ doped LCO crystals, the structure of the doped LCO crystals was determined by direct method and refined by Pawley method TOPAZ version programme using the single crystal X-ray data for pure LCO. The crystal data of LCO and Li doped LCO are presented in Table 1.

It is seen that both the pure and doped crystals crystallize in orthorhombic crystal system with $\mathrm{P} 2{ }_{1} 2_{1} 2_{1}$ space group. There are slight variations in the lattice parameters of the lithium doped crystal, compared to the pure one, due to the incorporation of $\mathrm{Li}^{+}$in the LCO crystal. Lithium doped L-citrulline oxalate has the same crystal structure and comparable lattice parameters as those of LCO.

\subsection{ICP AES Analysis}

The presence of lithium in the doped sample was confirmed from the inductively coupled plasma, atomic emission studies (ICPAES). From the measurements it is found that the concentration of lithium is $6.583 \mathrm{ppm}$ in the doped sample. The AES result (Table 2) shows that the metallic dopant is incorporated into the crystal lattice.

\subsection{FT-IR and Raman Spectral Studies}

The Fourier transform infrared (FT-IR) spectra were recorded for the pure and doped LCO crystals in the range $400-4000 \mathrm{~cm}^{-1}$, in order to qualitatively analyze the presence of functional groups. The recorded spectra are shown in Figure 3.

For saturated amines, the asymmetric $\mathrm{NH}_{2}$ stretch will give rise to a band between $3380 \mathrm{~cm}^{-1}$ and $3350 \mathrm{~cm}^{-1}$

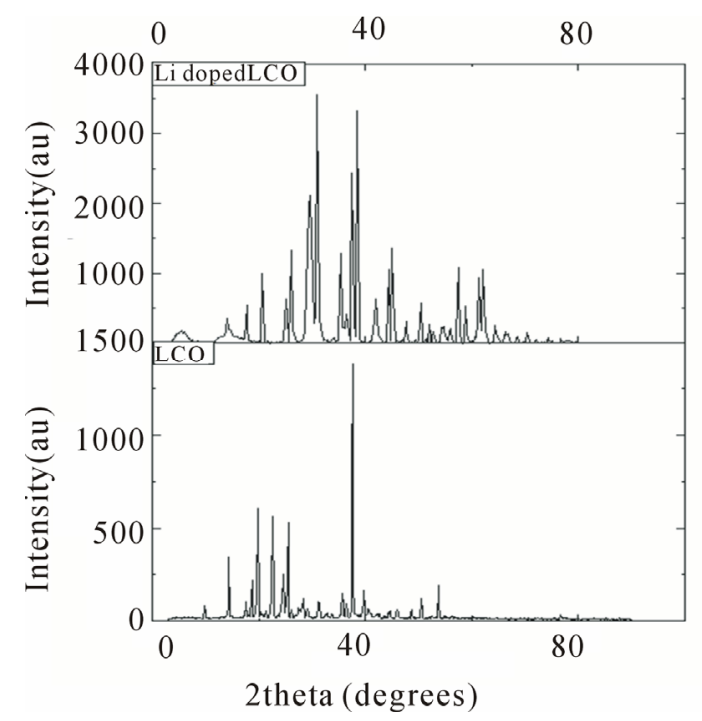

Figure 2. XRD pattern of pure and lithium doped L-citrulline oxalate crystals. 
Table 1. Crystal data of pure and $\mathrm{Li}^{+}$doped LCO crystals.

\begin{tabular}{ccc}
\hline Crystal parameters & Pure LCO & $\mathrm{Li}^{+}$doped LCO \\
\hline $\mathrm{a}(\AA)$ & $5.208(5)$ & 5.1963443 \\
$\mathrm{~b}(\AA)$ & $9.829(5)$ & 9.8636879 \\
$\mathrm{c}(\AA)$ & $23.879(5)$ & 23.8488366 \\
\hline
\end{tabular}

Table 2. Data of ICP atomic emission studies.

\begin{tabular}{ccc}
\hline Sample No. & Sample name & Element measured \\
\hline & & Li 6707 \\
1 & $\begin{array}{c}\text { Lithium doped } \\
\text { LCO }\end{array}$ & $6.583 \mathrm{ppm}$ \\
\hline
\end{tabular}

while the symmetric stretch will appear between 3310 $\mathrm{cm}^{-1}$ and $3280 \mathrm{~cm}^{-1}$ [14]. But the protonation of $\mathrm{NH}_{2}$ group can produce a shift in band position towards the lower wave number region. The $\mathrm{NH}^{3+}$ asymmetric and symmetric bending occur in the region around $1633 \mathrm{~cm}^{-1}$. The $\mathrm{NH}^{3+}$ rocking modes occur at wave numbers around $1100 \mathrm{~cm}^{-1}$ and the wagging $\mathrm{NH}$ vibrations around 720 $\mathrm{cm}^{-1}$. FT-IR vibrational spectral analysis establishes the existence of $\mathrm{NH}_{3}^{+}$group in the crystal confirming the protonation of the amino group for the formation of pure and doped L-citrulline oxalate. The IR spectrum recorded for doped crystals is similar to that of pure L-citrulline oxalate crystals and confirms the presence of all functional groups.

To analyze the presence of functional groups in the samples Raman spectra were also recorded for the pure and doped LCO in the range $50-3500 \mathrm{~cm}^{-1}$. The recorded spectra are shown in Figure 4. The $\mathrm{NH}_{3}^{+}$ stretching frequencies are found between $3100 \mathrm{~cm}^{-1}$ and $2600 \mathrm{~cm}^{-1}$ in the form of a broad strong band with multiple peaks. The $\mathrm{NH}_{3}^{+}$symmetric stretching occurs at $2985 \mathrm{~cm}^{-1}$ and for the doped one at $2977 \mathrm{~cm}^{-1}$. The $\mathrm{NH}_{3}^{+}$asymmetric and symmetric bending vibrations occur in the region $1641 \mathrm{~cm}^{-1}$ and $1528 \mathrm{~cm}^{-1}$. The $\mathrm{NH}_{3}^{+}$ parallel rocking modes are assigned to $1067 \mathrm{~cm}^{-1}$ for pure and to $1058 \mathrm{~cm}^{-1}$ for doped crystals in Raman spectra while the perpendicular modes to $994 \mathrm{~cm}^{-1}$ and $921 \mathrm{~cm}^{-1}$ respectively. The Raman vibrational spectral analysis establishes the existence of $\mathrm{NH}_{3}^{+}$group in the crystal confirming the protonation of the amino group for the formation of L-citrulline oxalate.

The asymmetric stretching bands at $2939 \mathrm{~cm}^{-1}$ and $2979 \mathrm{~cm}^{-1}$ in Raman spectra and the corresponding symmetric bands at $2893 \mathrm{~cm}^{-1}$ and $2897 \mathrm{~cm}^{-1}$ for the pure and doped crystals respectively denote the $\mathrm{CH}_{2}$ stretching bands for amino acids. The rocking $\mathrm{CH}_{2}$ band occur at $994 \mathrm{~cm}^{-1}$ and $992 \mathrm{~cm}^{-1}$. The sharp intense band at 1720 $\mathrm{cm}^{-1}$ and $1714 \mathrm{~cm}^{-1}$ in Raman spectra correspond to
$\mathrm{C}=\mathrm{O}$ vibrations. It is inferred from the peaks at 1315 $\mathrm{cm}^{-1}, 1431 \mathrm{~cm}^{-1}$ and $1608 \mathrm{~cm}^{-1}$ that they are due to the $\mathrm{C}=\mathrm{O}$ stretching of carboxylic group. The absence of peak at $1608 \mathrm{~cm}^{-1}$ for $\mathrm{C}=\mathrm{O}$ stretching in the Li doped-LCO may be due to the metal linkage with the carboxylic group. Also it can be seen that vibrational frequencies in both IR and Raman spectra are similar, confirming the noncentro symmetric nature of the crystals and highlight the prospects of LCO and the doped one as strong NLO materials.

\subsection{UV-Vis-NIR Absorption Studies}

The recorded absorption spectra of pure and lithiumdoped LCO crystals are shown in Figure 5. It is seen from the spectra that absorption is not observed between $400 \mathrm{~nm}$ to $800 \mathrm{~nm}$ (visible region). The absence of absorption of light in the visible region is an intrinsic property of all the amino acids [15]. The doped LCO crystals have better transparency window in the visible region

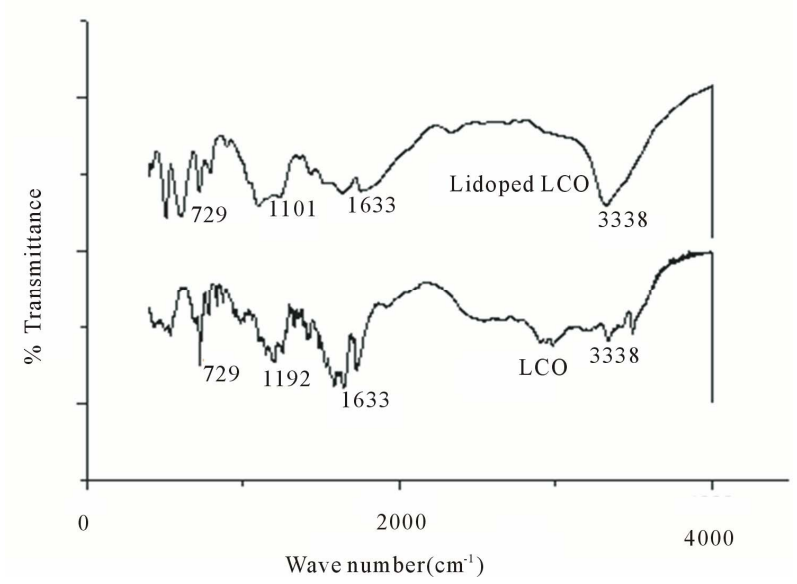

Figure 3. FTIR spectra of pure (LCO) and lithium doped (LCOL).

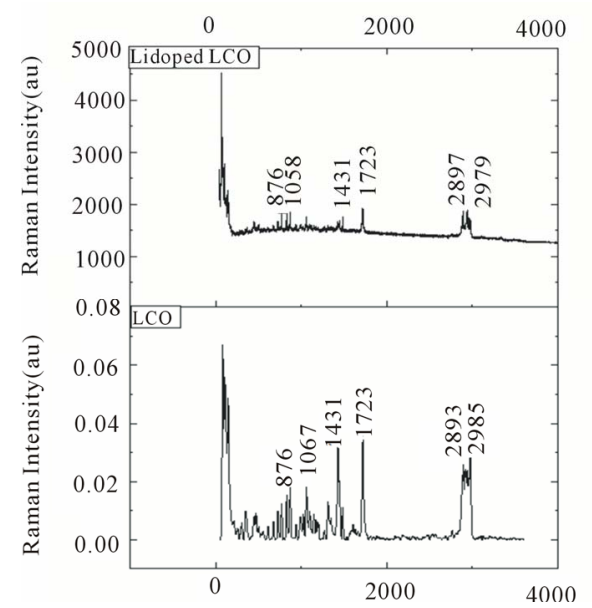

Figure 4. Raman spectrum of (a) pure LCO crystals; (b) Lithium doped LCO. 
which is about $50 \mathrm{~nm}$ wider than the pure sample. It can be concluded that the lithium dopant considerably improves the optical quality of L-citrulline oxalate crystals.

\subsection{TGA Analysis}

The thermo gravimetric analysis of pure and $\mathrm{Li}$ doped LCO crystals ware done in nitrogen atmosphere in the temperature range of $28^{\circ} \mathrm{C}-800^{\circ} \mathrm{C}$. Figure 6 shows the thermograms of pure and doped LCO. The decomposi tion of parent sample begins at $175^{\circ} \mathrm{C}$. But for lithium doped L-citrulline oxalate crystals, the decomposition starts at $225^{\circ} \mathrm{C}$. The increment in the decomposition temperature is evident for the doped crystals, suggesting that the substitution of lithium enhances the thermal stability.

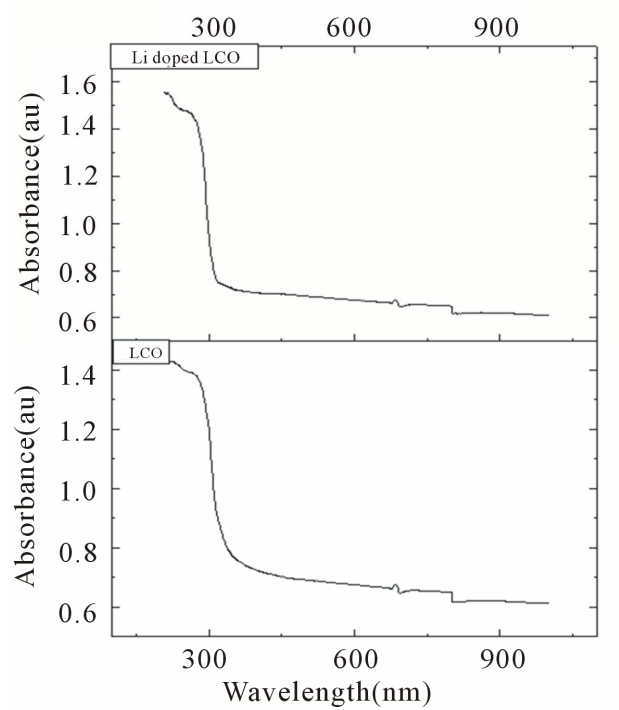

Figure 5. UV-Vis absorption spectra of pure (LCO) and $\mathrm{Li}^{+}$ doped (LCOL) samples.

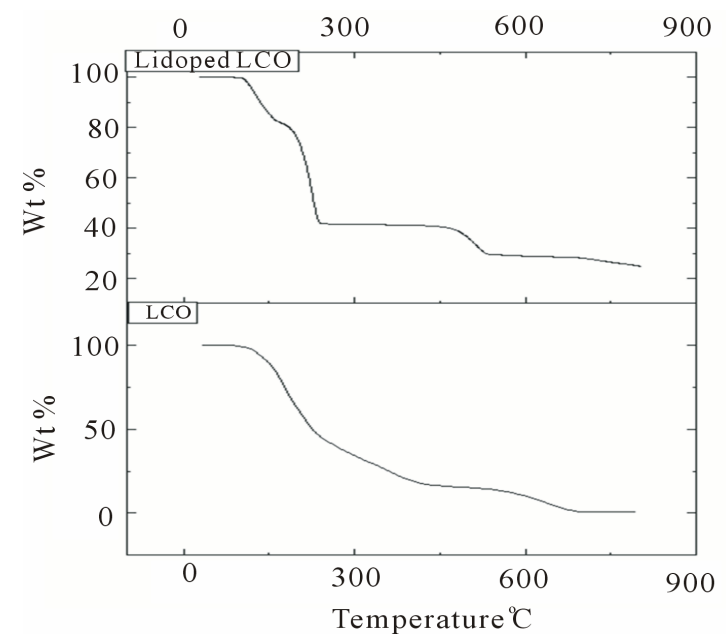

Figure 6. TGA curves for (a) pure and (b) $\mathrm{Li}^{+}$doped LCO samples.

\subsection{Second Harmonic Generation Efficiency}

SHG tests were carried out on the pure and doped LCO samples using the Nd:YAG Q-switched laser beam as the source. The SHG efficiency of grown samples was measured by using the Kurtz powder technique [16]. The fundamental $1064 \mathrm{~nm}$ beam from Q-switched Nd:YAG laser (Prolab 170 Quanta ray, pulse width $8 \mathrm{~ns}$, repetition rate $10 \mathrm{~Hz}$ ) was made to fall normally on the crystalline powder packed in a micro-capillary tube. For a laser input of $4.2 \mathrm{~mJ}$, the second harmonic signal (532 nm), 23 $\mathrm{mW}, 7 \mathrm{~mW}$ and $21 \mathrm{~mW}$ was obtained for KDP, pure LCO and $\mathrm{Li}^{+}$doped LCO. The SHG efficiency of LCO and that of doped LCO crystal is 0.3 and 0.9 times that of KDP respectively. Thus, the lithium metal has increased the SHG efficiency of pure LCO to a value almost comparable with KDP. Due to the presence of metal in the crystal lattice, there is an increase in polarizability of the molecule which tends to increase the SHG efficiency.

\section{Conclusion}

Good quality single crystals of pure and $\mathrm{Li}^{+}$doped Lcitrulline oxalate (LCO) were grown successfully by slow evaporation technique. The effect of doping with lithium in L-citrulline oxalate crystals is examined in this paper. The powder XRD studies confirm the structure of the doped crystals to be similar to the pure one. The presence of dopants has marginally altered the lattice parameters without affecting the basic structure of crystals. The thermal stability is found to be better for the doped samples. Also the UV absorption edge has shifted towards the blue region, increasing the transparency region from the IR to the middle of UV. The second harmonic generation efficiency for the doped sample has increased to a great extent making the doped crystal suitable for NLO applications. Good mechanical properties, excellent optical quality, moderate thermal stability, increase in SHG efficiency, make the $\mathrm{Li}^{+}$doped LCO crystals a strong candidate for NLO device fabrication

\section{Acknowledgements}

One of the authors Sreevalsa V. G. is grateful to the University Grants Commission for providing the Teacher Fellowship. The authors acknowledge stic, cusat for powder XRD and FTIR; LARPM, CIPET Bhuvaneswar for TGA and Prof. P. K. Das group IISc, Bangalore for providing facilities for taking SHG.

\section{REFERENCES}

[1] R. W. Boyd, "Nonlinear Optics," Academic Press, San Diego, 1992.

[2] D. S. Chemla and J. Zyss, "Nonlinear Optical Properties of Organic Molecules and Crystals," Vol. 1-2, Academic 
Press, New York, 1987.

[3] R. F. Belt, G. Gashurov and Y. S. Liu, "KTP as a Harmonic Generator of Nd:YAG Lasers," Laser Focus, Vol. 21, No. 10, 1985, pp. 110-124.

[4] R. S. Calark, "Getting the Laser Word to Subs," Photonics Spectra, Vol. 22, 1988, pp. 135-136.

[5] R. J. Gambino, “Optical Storage Disk Technology," Bulletin of Materials Research Society, Vol. 15, 1990, pp. 20-22.

[6] G. Rameshkumar, S. Gokul Raj, R. Mohan and R. Jayavel, "Influence of Isoelectric $\mathrm{pH}$ on the Growth Linear and Nonlinear Optical and Dielectric Properties of LThreonine Single Crystals," Crystal Growth and Design, Vol. 6, No. 6, 2006, pp. 1308-1310. doi: $10.1021 / \operatorname{cg} 050438 \mathrm{~g}$

[7] W. D. Cheng and J. X. Lu, "Certain Inorganic Borate Crystals as Excellent Second Order Nonlinear Optical Materials in the UV-Region," Chinese Journal of Structunal Chemistry, Vol. 16, 1997, pp. 81-85.

[8] M. Fujimoto and Y. Tomkiewicz, "EPR of Cationic Radicals Formed by UV Irradiation of $\mathrm{Cu}^{2+}$ Doped Amino Acid Crystals at $77^{\circ} \mathrm{K}$ : Glycine and Dimethylalanine," Journal of Chemical Physics, Vol. 56, No. 7, 1972, pp. 3317-3320. doi:10.1063/1.1677698

[9] B. S. Kumar, M. R. S. Kumar and K. R. Babu, "Growth and Characterization of Pure and Lithium Doped LAlanine Single Crystals for NLO Devices," Crystal Research and Technology, Vol. 43, No. 7, 2008, pp. 745750. doi:10.1002/crat.200711109

[10] P. P. Kumar, V. Manivannan, S. Tamilselvan, S. Senthil, V. A. Raj, P. Sagayaraj and J. Madhavan, "Growth and Characterization of a Pure and Doped Nonlinear Optical
L-Histidine Acetate Single Crystals," Optics Communications, Vol. 281, No. 10, 2008, pp. 2989-2995. doi:10.1016/i.optcom.2008.01.058

[11] P. P. Kumar, V. Manivannan, P. Sagayaraj and J. Madhavan, "Growth and Characterization of Pure and Doped NLO L-Arginine Acetate Single Crystals," Bulletin of Materials Science, Vol. 32, No. 4, 2009, pp. 431-435. doi:10.1007/s12034-009-0063-3

[12] V. G. Sreevalsa and S. Jayalekshmi, "Investigations on the Growth and Characterization of L-Citrulline Oxalate Monohydrate Single Crystal," Journal of Crystal Growth, Vol. 324, No. 1, 2011, pp. 172-176. doi:10.1016/j.jcrysgro.2011.03.017

[13] V. G. Sreevalsa and S. Jayalekshmi, "Studies on the Thermal, Optical and Dielectric Properties of a New Nonlinear Optical Crystal-L-Citrulline Oxalate Grown by Slow Evaporation Technique," Transactions of the Indian Institute of Metals, Vol. 64, No. 2, 2011, pp. 205208. doi:10.1007/s12666-011-0040-6

[14] D. Rajan Babu and D. Jayaraman, "Growth and Characterization of Non-Linear Optical L-Alanine Tetrafluoroborate (L-AlFB) Single Crystals," Journal of Crystal Growth, Vol. 245, No. 1-2, 2002, pp. 121-125.

[15] K. Ambujam, S. Selvakumar, D. P. Anand, G. Mohamed and P. Sagayaraj, "Crystal Growth, Optical, Mechanical and Electrical Properties of Organic NLO Material $\gamma$ Glycine," Crystal Research and Technology, Vol. 41, No. 7, 2006, pp. 671-677. doi:10.1002/crat.200510647

[16] S. K. Kurtz and T. T. Perry, "A Powder Technique for the Evaluation of Nonlinear Optical Materials," Journal of Applied Physics, Vol. 39, No. 8, 1968, pp. 3798-3813. doi:10.1063/1.1656857 